\title{
Dark matter blind spots at one-loop
}

\author{
Tao Han, ${ }^{a, b, c}$ Hongkai Liu, ${ }^{a}$ Satyanarayan Mukhopadhyay ${ }^{d}$ and Xing Wang ${ }^{a}$ \\ ${ }^{a}$ Department of Physics and Astronomy, University of Pittsburgh, \\ Pittsburgh, PA 15260, U.S.A. \\ ${ }^{b}$ Department of Physics, Tsinghua University, \\ Beijing, 100086, China \\ ${ }^{c}$ Collaborative Innovation Center of Quantum Matter, \\ Beijing, 100086, China \\ ${ }^{d}$ School of Physical Sciences, Indian Association for the Cultivation of Science, \\ Kolkata 700032, India \\ E-mail: than@pitt.edu, hol42@pitt.edu, tpsnm@iacs.res.in, \\ xiw77@pitt . edu
}

ABSTRACT: We evaluate the impact of one-loop electroweak corrections to the spinindependent dark matter (DM) scattering cross-section with nucleons $\left(\sigma_{\mathrm{SI}}\right)$, in models with a so-called blind spot for direct detection, where the leading-order prediction for the relevant DM coupling to the Higgs boson, and therefore $\sigma_{\mathrm{SI}}$, are vanishingly small. Adopting a simple illustrative scenario in which the DM state results from the mixing of electroweak singlet and doublet fermions, we compute the relevant higher order corrections to the scalar effective operator contributions to $\sigma_{\mathrm{SI}}$, stemming from both triangle and box diagrams involving the SM and dark sector fields. It is observed that in a significant region of the singlet-doublet model-space, the one-loop corrections "unblind" the tree-level blind spots and lead to detectable SI scattering rates at future multi-ton scale liquid Xenon experiments, with $\sigma_{\mathrm{SI}}$ reaching values up to a few times $10^{-47} \mathrm{~cm}^{2}$, for a weak scale DM with $\mathcal{O}(1)$ Yukawa couplings. Furthermore, we find that there always exists a new SI blind spot at the next-to-leading order, which is perturbatively shifted from the leading order one in the singlet-doublet mass parameters. For comparison, we also present the tree-level spin-dependent scattering cross-sections near the SI blind-spot region, that could lead to a larger signal. Our results can be mapped to the blind-spot scenario for bino-Higgsino DM in the MSSM, with other sfermions, the heavier Higgs boson, and the wino decoupled.

KeYwords: Beyond Standard Model, Supersymmetric Standard Model

ArXiv ePrint: 1810.04679 


\section{Contents}

1 Introduction $\quad 1$

2 Singlet-doublet dark matter and tree-level blind spot $\quad 3$

2.1 Spin-independent interaction 4

2.2 Spin-dependent interaction 5

3 Radiative corrections to DM-nucleon scattering 5

3.1 Computational framework 5

$\begin{array}{lll}3.2 & \text { Results } & 6\end{array}$

4 Direct detection: current constraints and future prospects $\quad 8$

4.1 Spin-independent scattering cross-sections at one-loop 8

$\begin{array}{lll}4.2 & \text { Tree-level spin-dependent scattering cross-sections } & 10\end{array}$

$\begin{array}{lll}5 & \text { Summary } & 12\end{array}$

$\begin{array}{ll}\text { A Details of on-shell renormalization scheme } & 13\end{array}$

B DM-nucleon scattering: computational framework $\quad 16$

$\begin{array}{ll}\text { C Mapping the singlet-doublet model to MSSM } & 17\end{array}$

\section{Introduction}

Weakly interacting massive particles (WIMP), a possible candidate for the dark matter $(\mathrm{DM})$ in the Universe, are being intensely searched for both in laboratory experiments and through a broad range of astrophysical probes [1,2]. Among the laboratory probes, the decades-long programme looking for signals of nuclear recoil is the primary one, with increasing levels of sensitivity to the DM-nucleon scattering rate, owing to both larger fiducial detector volumes, as well as the construction of ultra-low noise detectors [3-5]. The current level of experimental sensitivity therefore calls for increased accuracy of the theoretical predictions as well, in order to thoroughly probe interesting and well-motivated WIMP scenarios. This becomes especially important if the leading order predictions for these scattering cross-sections are negligibly small or even exactly zero either due to symmetry reasons or due to cancellations among different contributions to the relevant DM effective couplings. Next-to-leading order corrections then become important, and would constitute a benchmark for the near-future multi-ton scale liquid Xenon-based direct detection experiments, targeting at a DM-nucleon scattering cross-section below $10^{-47} \mathrm{~cm}^{2}$. 
A well-studied example of the above scenario where the one-loop contributions to the DM-nucleon scattering rate become important is DM belonging to a multiplet of the Standard Model (SM) weak interaction group SU(2) L $[6,7]$. For both real $\mathrm{SU}(2)_{\mathrm{L}}$ triplets with zero hypercharge (e.g., the wino in the minimal supersymmetric standard model, MSSM) and Majorana $\mathrm{SU}(2)_{\mathrm{L}}$ doublets (e.g., the Higgsino in the MSSM) the leading contribution to spin-independent (SI) scattering with nucleons appears at one-loop. In the former case, the SI cross-section with nucleon is only mildly sensitive to the DM mass and is obtained to be around $2.3 \times 10^{-47} \mathrm{~cm}^{2}$ in the limit $M_{\mathrm{DM}} \gg M_{W}$, including higher order corrections at next-to-leading order in $\alpha_{s}$ [8-12]. Therefore, these DM candidates are natural benchmark targets for multi-ton scale detectors. For Higgsino-like SU(2) L doublet Majorana fermions, the rate is further suppressed by two orders of magnitude, and the SI cross-section is around $10^{-49} \mathrm{~cm}^{2}$. Such cross-sections are below the irreducible neutrino floor [13, 14], thereby making necessary larger detector volumes and exposure time, as well as the development of directional detection methods [15, 16].

While for the pure $\mathrm{SU}(2)_{\mathrm{L}}$ multiplets discussed above the tree-level SI scattering rates are absent due to symmetry reasons, there are other scenarios in which very small tree-level rates are obtained due to cancellations of different contributions to the relevant effective couplings. For example, if the neutral components of different $\mathrm{SU}(2)_{\mathrm{L}}$ multiplets mix after electroweak symmetry breaking, generically there are regions of parameter space where the effective coupling to the Higgs boson(s), which determines the leading contribution to the SI scattering rate, either becomes small or even vanishes, a scenario dubbed as "blind spots" for DM direct detection [17-27]. While the particular values and relations of the theory parameters that result in the blind spots may not have any deeper theoretical implications, or may even be viewed as a fine-tuning to a special hypersurface within the parameter space, they do characterize a distinctive class of phenomena that need to be scrutinized. Such blind spots for DM-nucleon scattering therefore present us with another context in which the higher-order electroweak corrections, involving states from both the dark matter and the SM sectors in the loop amplitudes, are important to evaluate in order to quantify its detectability. In this paper, we compute the one-loop corrections to DM-nucleon scattering processes near such blind spots, and assess their implications for different direct detection probes.

As an example scenario, which represents all the features of more involved models such as the bino-Higgsino mixed DM in the MSSM [1], we begin by studying a DM model with mixing between an $\mathrm{SU}(2)_{\mathrm{L}} \times \mathrm{U}(1)_{\mathrm{Y}}$ singlet fermion and the neutral components of two $\mathrm{SU}(2)_{\mathrm{L}}$ doublet fermions $[25,28,29]$. The details of this simplified model and the appearance of tree-level blind spots are reviewed in section 2. We then systematically evaluate the impact of the one-loop corrections for the SI scattering rates near the blind spots in the singlet-doublet model, after defining an on-shell renormalization procedure for the dark matter sector. The computational framework and the results of the oneloop corrections are discussed in section 3, while the details of the on-shell renormalization scheme adopted are summarized in appendix A. In section 4 we utilize these one-loop results to find out the prospects of observing DM-nucleon scattering near the tree-level blind spots. In this section, we also compare the prospects for probing the one-loop SI rates with the 
reach from the tree-level spin-dependent (SD) DM-nucleon scattering searches. We provide a summary of our study in section 5 . We also briefly review the computational framework adopted in this paper for SI and SD DM-nucleon scattering in appendix B, and the mapping of the singlet-doublet model parameters to the case for MSSM bino-Higgsino mixed DM scenario in appendix $\mathrm{C}$.

\section{Singlet-doublet dark matter and tree-level blind spot}

To understand the appearance of blind spots for DM direct detection, it is instructive to consider a simple model, in which the DM candidate is a linear combination of an electroweak singlet Majorana fermion $\chi_{S}$, and the neutral components of two $\mathrm{SU}(2)_{\mathrm{L}}$ doublet states $\chi_{D 1}$ and $\chi_{D 2}$, with hypercharge $+1 / 2$ and $-1 / 2$, respectively $[17,18,25]$,

$$
\chi_{D 1}=\left(\chi_{1}^{+}, \chi_{1}^{0}\right)^{\top} \quad \text { and } \quad \chi_{D 2}=\left(\chi_{2}^{0}, \chi_{2}^{-}\right)^{\top} .
$$

The mixing between the singlet and the neutral components of the doublet states occurs after electroweak symmetry breaking. Such a scenario can appear in beyond-the-standardmodel constructions such as the MSSM, in which the singlet state is the bino, and the two doublet states correspond to the two Higgsinos. In the MSSM some of the couplings of these states with the SM sector are determined by gauge symmetry and supersymmetry, and therefore the results of the singlet-doublet model can be mapped to the MSSM case, as long as all the sfermions, heavy scalars and wino are decoupled.

In order to have a stable DM candidate, we impose an additional $Z_{2}$ symmetry, under which the DM sector states are odd, and all the SM sector states are even. Thus, the lightest neutral state in the dark sector is the DM candidate, where the mass spectrum and Yukawa couplings of the dark sector particles are determined by the following Lagrangian

$$
\mathscr{L}_{Y}=-\left(\frac{1}{2} M_{S} \chi_{S} \chi_{S}+M_{D} \chi_{D 1} \cdot \chi_{D 2}-y_{1} \chi_{S} \chi_{D 1} \cdot \widetilde{H}-y_{2} \chi_{S} \chi_{D 2} \cdot H\right)+\text { h.c. }
$$

where $H=\left(\phi^{+},(v+h+i \eta) / \sqrt{2}\right)^{\top}$ is the SM Higgs doublet, with a vacuum expectation value $v=246 \mathrm{GeV}$, while $\widetilde{H}=i \sigma_{2} H^{*}$. The dot products in eq. (2.2) indicate the contraction of $\mathrm{SU}(2)_{\mathrm{L}}$ indices to form a singlet.

We see that the mass spectrum is determined by four free parameters, namely, $M_{D}, M_{S}$, $y_{1}$ and $y_{2}$. By re-defining the fields $\chi_{D 1}, \chi_{D 2}$, and $\chi_{S}$, we can make three of them positive, chosen to be $y_{1}, y_{2}$, and $M_{S}$. For simplicity, we do not include any possible CP violation in the DM sector, and restrict to real values of $M_{D}$ only. After electroweak symmetry breaking, the neutral components of the doublet and singlet dark fermions mix, and the mass matrix of neutral dark sector in the gauge basis $\chi^{0}=\left(\chi_{S},-\chi_{2}^{0}, \chi_{1}^{0}\right)^{\top}$ is given by

$$
\mathbf{M}_{\mathbf{N}}=\left(\begin{array}{ccc}
M_{S} & \frac{y v \cos \beta}{\sqrt{2}} & \frac{y v \sin \beta}{\sqrt{2}} \\
\frac{y v \cos \beta}{\sqrt{2}} & 0 & M_{D} \\
\frac{y v \sin \beta}{\sqrt{2}} & M_{D} & 0
\end{array}\right),
$$

where $\tan \beta=y_{1} / y_{2}$, with $y_{1}=y \sin \beta$ and $y_{2}=y \cos \beta$. 


\subsection{Spin-independent interaction}

The dominant contribution to SI direct detection cross-section stems from the Higgs boson exchange diagram, and we obtain the tree-level DM-Higgs coupling using the low energy theorem

$$
C_{h \widetilde{\chi}_{1}^{0} \widetilde{\chi}_{1}^{0}}^{0}=\frac{1}{2} \frac{\partial M_{\widetilde{\chi}_{1}^{0}}(v)}{\partial v}=\frac{y^{2} v\left[M_{D} \sin (2 \beta)+M_{\widetilde{\chi}_{1}^{0}}\right]}{6 M_{\widetilde{\chi}_{1}^{0}}^{2}-4 M_{\widetilde{\chi}_{1}^{0}} M_{S}-2 M_{D}^{2}-y^{2} v^{2}},
$$

where $\tilde{\chi}_{1}^{0}$ is the lightest neutral mass eigenstate. In the limit of vanishing momentum transfer relevant for nuclear-recoil experiments, the SI direct detection rate is fixed by the Wilson coefficient $f_{q}$ of the operator $m_{q} \overline{\widetilde{\chi}_{1}^{0}} \widetilde{\chi}_{1}^{0} \bar{q} q$. The $t$-channel Higgs exchange process leads to the following isospin-conserving Wilson coefficient for interactions with up-type and down-type quarks

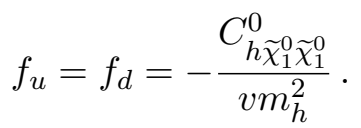

There is an additional effective coupling to a pair of gluons in the nucleon, which is obtained on integrating out the heavy quarks coupled to the Higgs propagator, and the corresponding Wilson coefficient is given as [1]

$$
f_{G}=-\frac{1}{12} \sum_{q=c, b, t} f_{q} .
$$

Combining the quark and the gluon contributions, we obtain the effective coupling of the DM state to nucleons, $f_{N} \overline{\widetilde{\chi}_{1}^{0}} \widetilde{\chi}_{1}^{0} \bar{N} N$, with

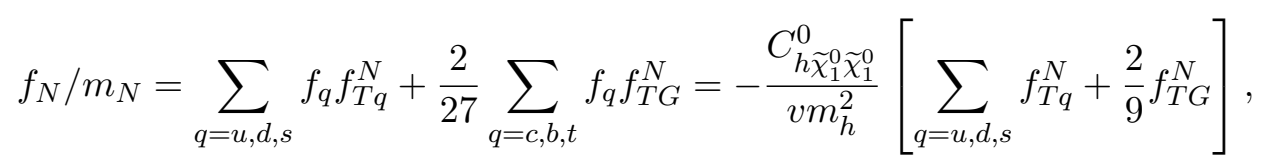

where $f_{T q}^{N}$ and $f_{T G}^{N}$ are the mass-fraction parameters of the quarks and the gluon in the nucleon $N$, respectively, and $f_{T G}^{N} \equiv 1-\sum_{q=u, d, s} f_{T q}^{N}$. We have summarized the additional details in the computation of DM-nucleon scattering in appendix B.

Thus, we see from the above discussion that at the leading order, the SI DM-nucleon scattering rate via the Higgs boson exchange would vanish if the mass and Yukawa coupling parameters satisfy the following blind-spot condition $[17,18,25]$

$$
M_{D} \sin (2 \beta)+M_{\widetilde{\chi}_{1}^{0}}=0 .
$$

For our choice of the phases of the mass and Yukawa coupling parameters, we see that the blind-spot condition can be satisfied for $M_{D}<0$. For the specific choice of parameters that satisfy the blind-spot condition, since the coupling of the DM mass eigenstate to the Higgs boson is zero, the physical mass of the DM state is either $M_{S}$ or $M_{D}$, depending upon the hierarchy. Thus the two possibilities are

1. $M_{\widetilde{\chi}_{1}^{0}}=M_{S},-M_{D}>M_{S}, \sin (2 \beta)=M_{S} /\left(-M_{D}\right)$,

2. $M_{\widetilde{\chi}_{1}^{0}}=-M_{D},-M_{D}<\left(M_{S}+\sqrt{M_{S}^{2}+(y v)^{2}}\right) / 2, \tan \beta=1$. 
While the first possibility leads to an SI blind spot, the second one implies a blind spot for both SI and SD scattering. For our subsequent analyses, we take up the first case as an illustration.

\subsection{Spin-dependent interaction}

In the singlet-doublet model, the spin-dependent interaction of DM with the nucleon is determined by the gauge interaction of the doublet components with the $Z$-boson. The relevant interaction Lagrangian is given in terms of the gauge eigenstates by

$$
\mathscr{L}_{\mathrm{int}}=-\frac{e}{2 \cos \theta_{W} \sin \theta_{W}}\left[\left(\chi_{1}^{0}\right)^{\dagger} \sigma_{-}^{\mu} \chi_{1}^{0}-\left(\chi_{2}^{0}\right)^{\dagger} \sigma_{-}^{\mu} \chi_{2}^{0}\right] Z_{\mu},
$$

where $\theta_{W}$ is the Weinberg angle. Thus the axial-vector coupling of the DM state to the $Z$-boson, which leads to the spin-dependent interaction with nucleons is obtained to be

$$
C_{Z \widetilde{\chi}_{1}^{0} \widetilde{\chi}_{1}^{0}}^{0}=\frac{e}{2 s_{W} c_{W}}\left(U_{21}^{2}-U_{31}^{2}\right),
$$

where $s_{W}=\sin \theta_{W}, c_{W}=\cos \theta_{W}$, and the mixing matrix in the neutral dark sector is defined by

$$
\widetilde{\chi}^{0}=U^{\dagger} \chi^{0}
$$

with the mass eigenstates $\widetilde{\chi}^{0}=\left(\widetilde{\chi}_{1}^{0}, \widetilde{\chi}_{2}^{0}, \widetilde{\chi}_{3}^{0}\right)^{\top}$. Therefore, the Wilson coefficient of the relevant low-energy effective interaction $\widetilde{\chi}_{1}^{0} \gamma^{\mu} \gamma^{5} \widetilde{\chi}_{1}^{0} \bar{q} \gamma_{\mu} \gamma^{5} q$ is found to be (please see appendix B for further details on the standard formalism adopted)

$$
d_{u}=\frac{-e^{2}\left(U_{21}^{2}-U_{31}^{2}\right)}{8 M_{Z}^{2} s_{W}^{2} c_{W}^{2}}=-d_{d}
$$

\section{Radiative corrections to DM-nucleon scattering}

We now turn to the electroweak radiative corrections to the spin-independent DM direct detection rate near the tree-level blind spots. Since the SI scattering rates are vanishingly small around this region of mass and coupling parameters, the next-to-leading order (NLO) corrections are expected to play an important role in determining the detectability of such DM model-space. Furthermore, as we will see in the following, there also appears a new blind spot at NLO order, at a shifted parameter region compared to the tree-level one.

\subsection{Computational framework}

In addition to the interaction Lagrangians described in eqs. (2.2) and (2.9), the following additional interaction terms (in the gauge basis) involving the charged components of the DM doublets and the weak bosons enter the computation of the radiative corrections

$$
\mathscr{L}_{\text {int }}=\frac{e}{\sqrt{2} s_{W}}\left[\left(\left(\chi_{1}^{0}\right)^{\dagger} \sigma_{-}^{\mu} \chi_{1}^{+}+\left(\chi_{2}^{-}\right)^{\dagger} \sigma_{-}^{\mu} \chi_{2}^{0}\right) W_{\mu}^{-}+\text {h.c. }\right] .
$$




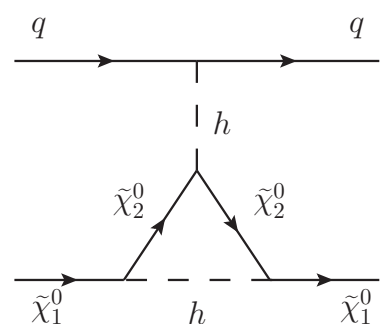

(a)

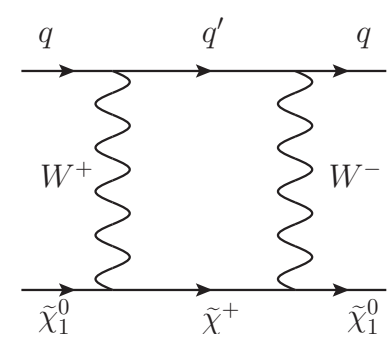

(b)

Figure 1. Representative Feynman diagrams contributing to DM-quark spin-independent scattering.

There are two different amplitudes contributing to the NLO electroweak corrections to DM-nucleon scattering, with representative Feynman diagrams depicted in figure 1. The first one stems from the one-loop vertex corrections to the Higgs-DM coupling, as shown in figure 1a, while the second one is given by the box diagrams shown in figure 1b. Since the triangle diagrams are ultraviolet (UV) divergent, we need to renormalize the relevant mass, mixing and coupling parameters. We have adopted the on-shell renormalization scheme for the dark matter sector, the details of which are described in appendix A.

In addition to the class of diagrams represented in figure 1, there are other sets of diagrams entering the NLO electroweak corrections to the same process. These involve the Higgs self-energy corrections and the vertex corrections to the quark Yukawa couplings. However, the contribution of these latter diagrams to the DM-quark effective vertex is proportional to the tree-level DM-Higgs coupling, which is vanishingly small near the treelevel blind-spot region of our interest. We therefore focus on the diagrams in figure 1 for our computation, which constitute a UV-finite subset.

We have generated the relevant Feynman diagrams and the corresponding matrix elements using FeynArts [30], which are then passed onto FeynCalc [31, 32] to perform the Passarino-Veltman reduction of the one-loop integrals. We have used Collier [33-36] for the numerical evaluation of the one-loop scalar integrals. We have adopted the Feynman gauge for our computations.

\section{$3.2 \quad$ Results}

The contribution to the effective DM-quark interaction from the vertex corrections represented by the triangle diagrams in figure $1 \mathrm{a}, f_{N}^{\text {tri }}$, has the same form as the tree-level $t$-channel Higgs exchange vertex, with the Higgs-DM coupling $C_{h \widetilde{\chi}_{1}^{0} \widetilde{\chi}_{1}^{0}}^{0}$ replaced by its oneloop counterpart $C_{h \widetilde{\chi}_{1}^{0} \widetilde{\chi}_{1}^{0}}^{\text {tri }}$

$$
f_{N}^{\mathrm{tri}} / M_{N}=-\frac{C_{h \widetilde{\chi}_{1}^{0} \widetilde{\chi}_{1}^{0}}^{\mathrm{tri}}}{v m_{h}^{2}}\left(\sum_{q=u, d, s} f_{T q}+\frac{2}{9} f_{T G}\right) .
$$

The box diagrams shown in figure $1 \mathrm{~b}$ also induce corrections to the Wilson coefficient of the operator $\widetilde{\chi}_{1}^{0} \widetilde{\chi}_{1}^{0} \bar{q} q$, denoted as $C_{q}^{\text {box }}$, which are not universal for different flavors, and 


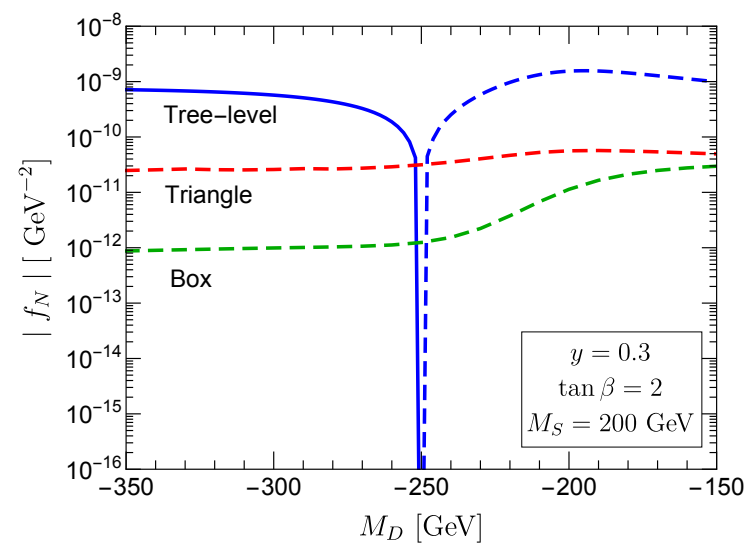

(a)

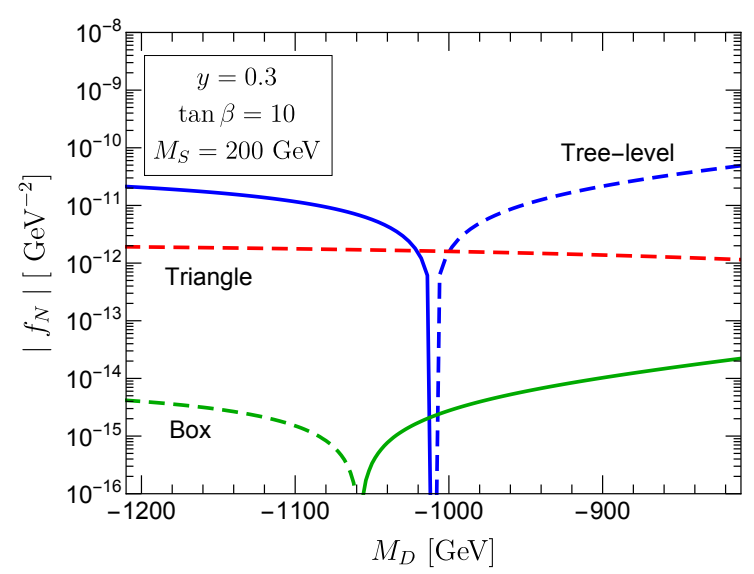

(c)

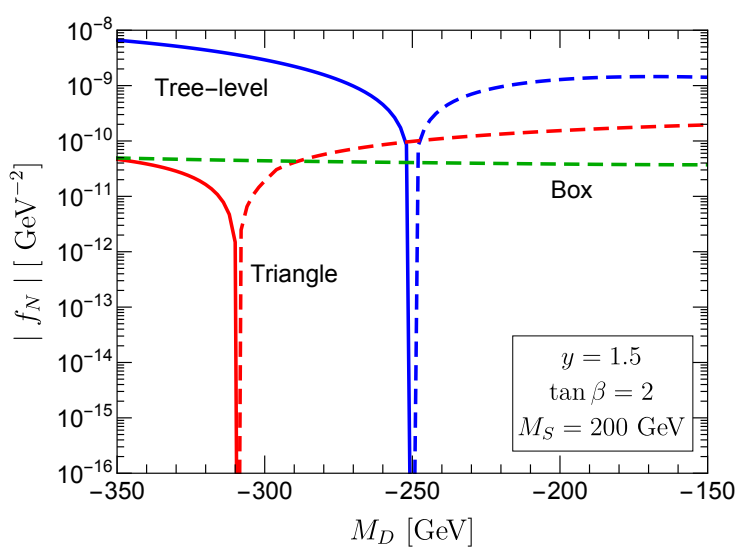

(b)

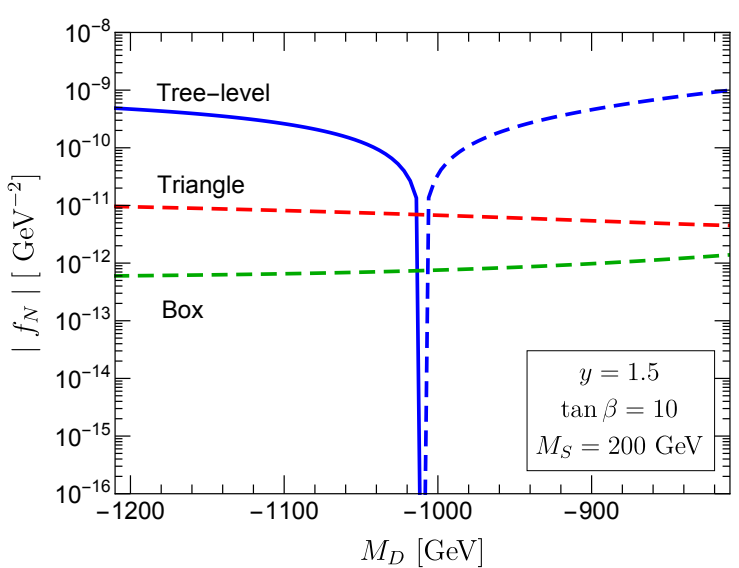

(d)

Figure 2. Contributions to the absolute value of $f_{N}$ as a function of $M_{D}$, from tree-level diagrams (blue), one-loop triangle diagrams (red), and one-loop box diagrams (green). The dashed lines indicate negative values of $f_{N}$. The value of the singlet dark fermion mass is fixed as $M_{S}=200 \mathrm{GeV}$, with $\tan \beta=2$ (upper panels) and $\tan \beta=10$ (lower panels), for representative values of $y=0.3$ (left columns) and $y=1.5$ (right columns).

lead to the following corrections to the DM-nucleon effective scalar coupling:

$$
f_{N}^{\text {box }} / M_{N}=\sum_{q=u, d, s} \frac{C_{q}^{\text {box }}}{m_{q}} f_{T q}+\frac{2}{27} f_{T G} \sum_{q=c, b, t} \frac{C_{q}^{\text {box }}}{m_{q}} .
$$

The "long-distance" contribution $[10,12]$ induced by the loop-diagrams to DM-gluon interaction is given by the second term in eq. (3.3).

We show the resulting magnitudes of the tree-level $f_{N}^{\text {tree }}$, the triangle diagram $f_{N}^{\text {tri }}$, and the box diagram $f_{N}^{\text {box }}$ contributions as a function of $M_{D}$ in figure 2 , where we have adopted the Feynman gauge for our computations. The results are shown for $M_{S}=200 \mathrm{GeV}$ with various values of $y$ and $\tan \beta$. Here, dashed lines have been used to indicate negative values of the Wilson coefficients. We note several interesting features in figure 2. First of all, although the tree-level contribution naturally dominates in the parameter region away from 
the blind spot, near the blind spot it decreases dramatically. The one-loop contribution, especially from the triangle diagrams, therefore gives rise to the leading contribution in this region. Secondly, away from the blind spot, the one-loop electroweak effects are still appreciable. For example, we see in figures $2 \mathrm{~b}$ and $2 \mathrm{c}$ that the contributions from the triangle diagrams considered can shift the tree-level results by up to $10 \%$. Third, the box diagram contribution can be comparable to the triangles in certain regions of parameter space. Fourth, there are values of parameters around which the triangle and the box contributions can change sign individually, and therefore have their own blind spots, as seen in figures $2 \mathrm{~b}$ and $2 \mathrm{c}$.

Most importantly, the full amplitude, which is a coherent sum of all the diagrams, always shows a new blind spot at the NLO level, perturbatively shifted from the tree-level blind spot. We quantify this shift by introducing a mass parameter difference

$$
\delta M_{D}=M_{D}^{(0)}-M_{D}^{(1)},
$$

which is the difference between the tree-level blind spot $M_{D}^{(0)}=-M_{\widetilde{\chi}_{1}^{0}} / \sin 2 \beta$ and the new blind spot $M_{D}^{(1)}$ obtained at NLO, on including the one-loop corrections. This variation in $\delta M_{D}$ is shown in figure 3 as a function of $M_{S}$. The amount of the shift in the values of $M_{D}$ is almost linearly proportional to the value of $M_{S}$ as seen in figures $3 \mathrm{a}$ and 3c. The results are shown for two values of the coupling $y=0.3$ (red) and $y=1.5$ (blue), with $\tan \beta=2$ (upper panels) and $\tan \beta=10$ (lower panels). As we can see from this figure, the shift is larger for large values of $\tan \beta$ and small values of $y$. We also show the ratio $\delta M_{D} / M_{D}^{(0)}$ in figures $3 \mathrm{~b}$ and $3 \mathrm{~d}$ (the right panels), and it can be around $\mathcal{O}(1 \%)$ for small values of $y$.

We note that the red curves in figures $3 \mathrm{a}$ and $3 \mathrm{~b}$, with $y=0.3$ and $\tan \beta=2$, exhibit two cusps at $M_{S} \simeq 330 \mathrm{GeV}$ and $470 \mathrm{GeV}$. These are due to the opening of new thresholds where the decays $\widetilde{\chi}_{2,3}^{0} \rightarrow \widetilde{\chi}_{1}^{0} Z$ and $\widetilde{\chi}_{2,3}^{0} \rightarrow \widetilde{\chi}_{1}^{0} h$, respectively, become kinematically accessible. ${ }^{1}$ On the other hand, the blue curves in figures $3 \mathrm{a}$ and $3 \mathrm{~b}$, and all the curves in figures $3 \mathrm{c}$ and $3 \mathrm{~d}$ do not have such cusps, as the decay channels $\widetilde{\chi}_{2,3}^{0} \rightarrow \widetilde{\chi}_{1}^{0} Z$ and $\widetilde{\chi}_{2,3}^{0} \rightarrow \widetilde{\chi}_{1}^{0} h$ are always allowed in the relevant parameter regions.

\section{Direct detection: current constraints and future prospects}

We now apply the results of the previous section to estimate the reach of ongoing and future direct detection experiments in the singlet-doublet model parameter space near the tree-level blind spot region. After discussing the NLO contribution to the spin-independent scattering, we also show the LO estimate for the reach of spin-dependent scattering experiments for comparison.

\subsection{Spin-independent scattering cross-sections at one-loop}

In this section, we focus on the parameter region for the tree-level SI blind spot, where the NLO corrections are most impactful in extending the reach of SI direct detection probes. For a fixed value of $\tan \beta$, this then leads to a two-dimensional parameter space of interest,

\footnotetext{
${ }^{1}$ The masses of $\widetilde{\chi}_{2}^{0}$ and $\widetilde{\chi}_{3}^{0}$ are nearly degenerate close to the tree-level blind spot parameter region.
} 


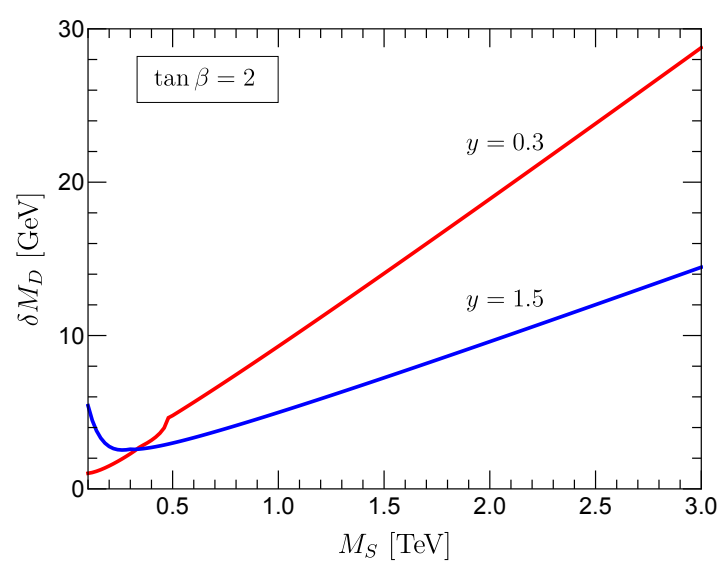

(a)

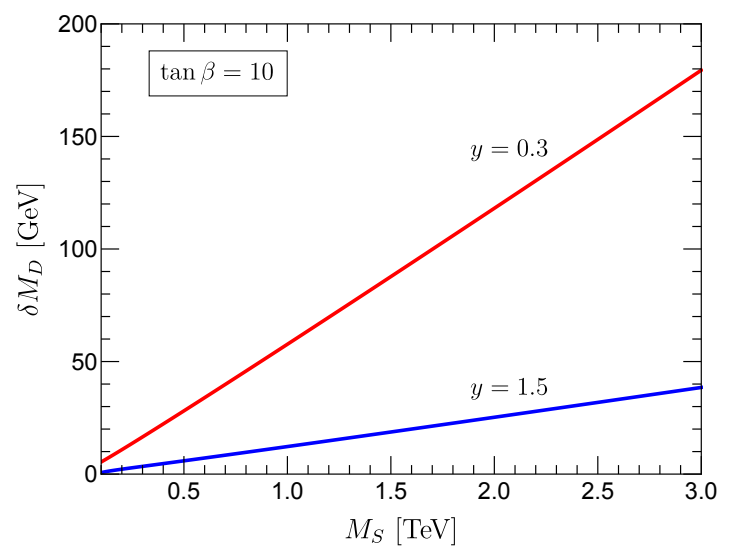

(c)

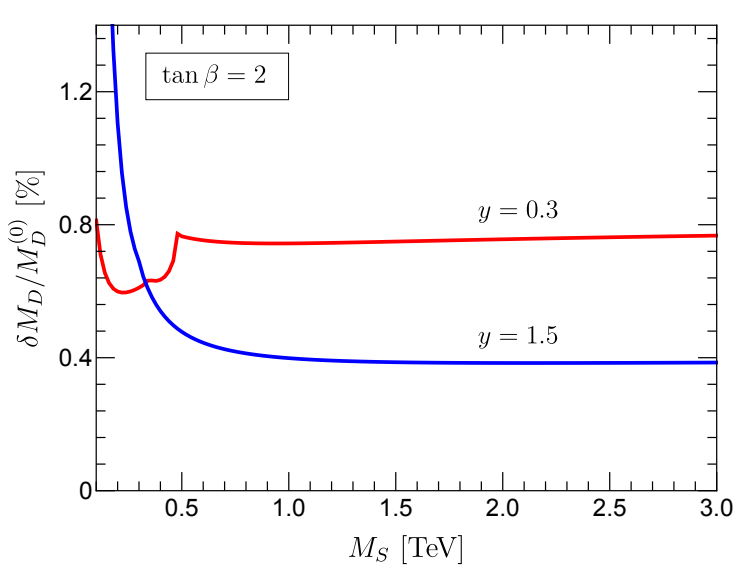

(b)

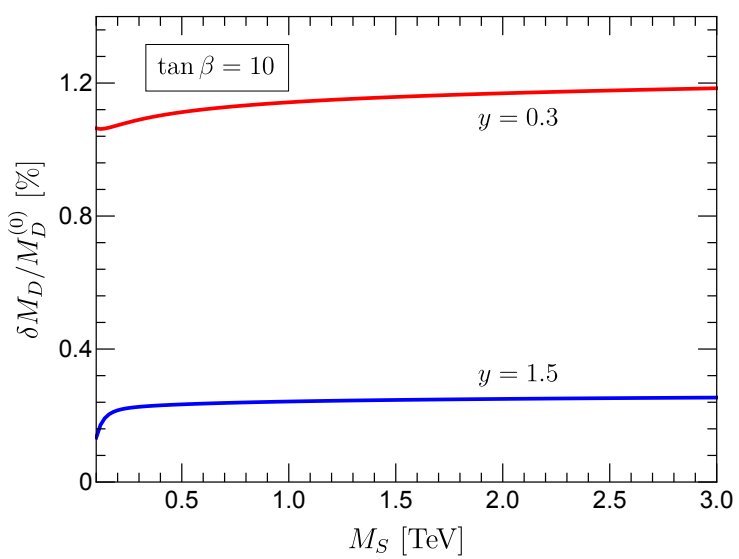

(d)

Figure 3. Shift in the position of the blind spot $\delta M_{D}$ versus $M_{S}$, with $\delta M_{D}=M_{D}^{(0)}-M_{D}^{(1)}$. The results are shown for two values of the coupling $y=0.3$ (red) and $y=1.5$ (blue), with $\tan \beta=2$ (upper panels) and $\tan \beta=10$ (lower panels). We also show the ratio $\delta M_{D} / M_{D}^{(0)}$ in the right panels.

that of the DM mass $\left(M_{\widetilde{\chi}_{1}^{0}}\right)$ and Yukawa coupling $y$ plane. The value of $M_{D}$, for each $M_{\widetilde{\chi}_{1}^{0}}$, is fixed to be $-M_{\widetilde{\chi}_{1}^{0}} / \sin (2 \beta)$ as given by the blind-spot condition in eq. (2.8).

In figure 4 , we show the contours of SI DM-nucleon scattering cross-section, $\sigma_{\mathrm{SI}}$, in the $y-M_{\widetilde{\chi}_{1}^{0}}$ plane, for values of $\tan \beta=2$ (left) and $\tan \beta=10$ (right). As we can see, for $\tan \beta=2, \sigma_{\mathrm{SI}}$ takes values in the range of about $10^{-47} \mathrm{~cm}^{2}$ to $10^{-50} \mathrm{~cm}^{2}$, for $M_{\widetilde{\chi}_{1}^{0}}$ values in the interval $100 \mathrm{GeV}-2 \mathrm{TeV}$, and coupling coefficient $y$ in the range $0.3-1.5$. For a given coupling, the cross-section decreases with increasing DM mass, and the future projection from the LZ experiment [38] (blue shaded region) is expected to probe a DM mass upto about $500 \mathrm{GeV}$ (blue shaded region in figure 4), for the above range of $y$, assuming the DM candidate to saturate the observed relic density. This reach can be further extended by the DARWIN experiment [39] (red shaded region), which can probe DM masses of upto $1250 \mathrm{GeV}$ for the same range of coupling values. For higher values of $\tan \beta$, as seen with $\tan \beta=10$ in the right panel of figure 4 , the expected cross-section is smaller due to the 


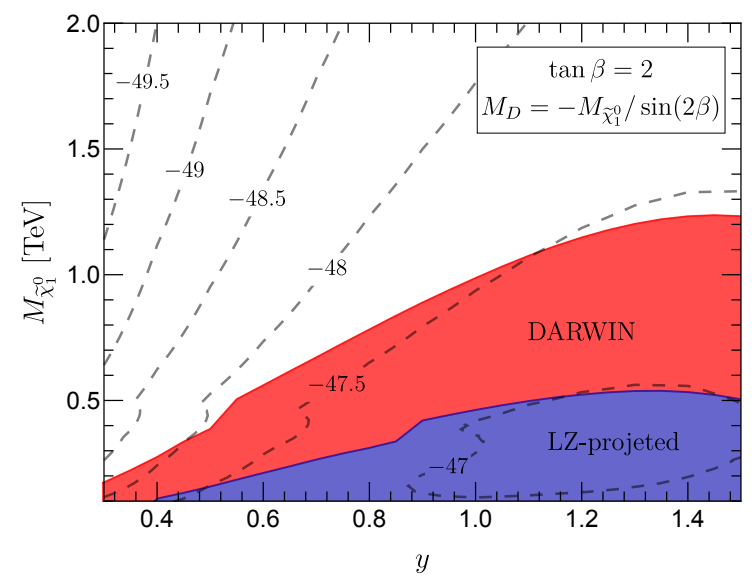

(a)

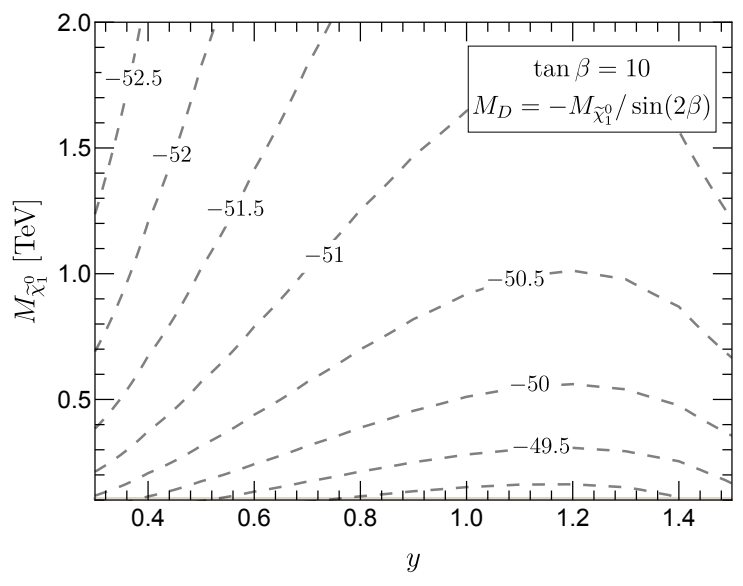

(b)

Figure 4. Spin-independent DM-nucleon scattering cross-section $\left(\sigma_{\mathrm{SI}}\right)$ in the DM mass $\left(M_{\widetilde{\chi}_{1}^{0}}\right)-$ Yukawa coupling $(y)$ plane, with $M_{D}$ fixed by the blind-spot condition. The results are shown for $\tan \beta=2$ (left panel) and $\tan \beta=10$ (right panel). The contours represent lines with fixed values of $\log _{10} \sigma^{\mathrm{SI}}$, with $\sigma_{\mathrm{SI}}$ expressed in $\mathrm{cm}^{2}$ units. The projected reach of the LZ [38] (blue shaded) and DARWIN [39] (red shaded) experiments are also shown, with the DM candidate assumed to saturate the observed relic density.

suppression from smaller mixing angles, with a maximum of around $10^{-49} \mathrm{~cm}^{2}$, which may not be accessible to DARWIN. Thus, the small $\tan \beta$ scenario leads to similar $\sigma_{\mathrm{SI}}$ as in the case of wino-like real triplet DM, as discussed in the introduction, while the intermediate $\tan \beta$ scenario predicts cross-sections similar to the case of Majorana Higgsino-like doublets.

\subsection{Tree-level spin-dependent scattering cross-sections}

In the spin-independent (SI) blind-spot region considered above, the effective coupling of the DM mass eigenstate to the Higgs boson vanishes. On the other hand, the spindependent (SD) scattering rate, which is determined at the tree level by the DM- $Z$-boson coupling, can have an appreciable rate for the same set of model parameters. In general, though the experimental sensitivity of SD scattering is weaker than that of SI scattering, near the blind spot they might have comparable reach [17, 26, 40], since the SI rates appear only at NLO.

We show the spin-dependent scattering cross-sections, $\sigma_{\mathrm{SD}}^{p}$ for proton and $\sigma_{\mathrm{SD}}^{n}$ for neutron, in figure 5 in the $y-M_{\widetilde{\chi}_{1}^{0}}$ plane, with all other parameters and conditions being the same as in figure $4 .^{2}$ The corresponding cross-sections are in the range of $10^{-38}-10^{-43} \mathrm{~cm}^{2}$ for $\tan \beta=2$, and around an order of magnitude lower for $\tan \beta=10$, in the parameter space studied. The reach from the current PICO-60 experiment [41-43] (blue shaded region) and the future projections from the LZ experiment [38] (red shaded region) are also shown. For $\tan \beta=2$, the reach from PICO-60 is upto about $M_{\widetilde{\chi}_{1}^{0}}=840 \mathrm{GeV}$, while

\footnotetext{
${ }^{2}$ We note that, since the parametric shift of the blind spot from tree-level to NLO is only of $\mathcal{O}(1 \%)$, the difference between SD cross-sections at the tree-level and NLO SI blind spots are negligible.
} 


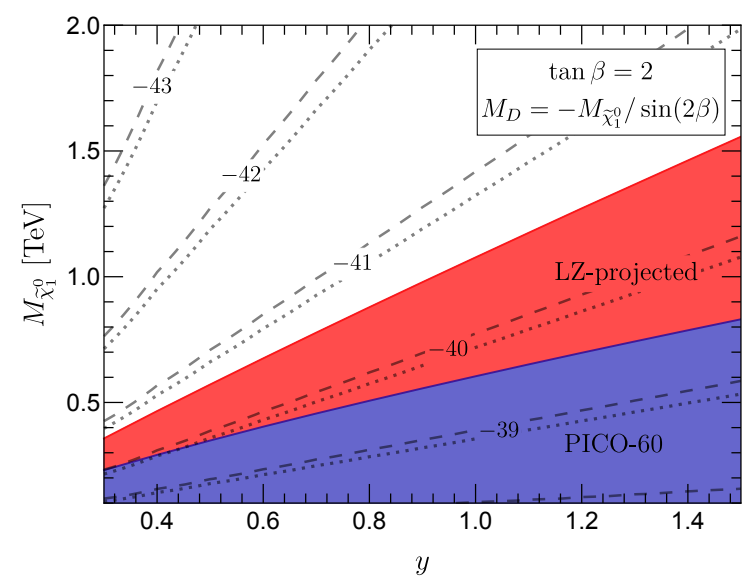

(a)

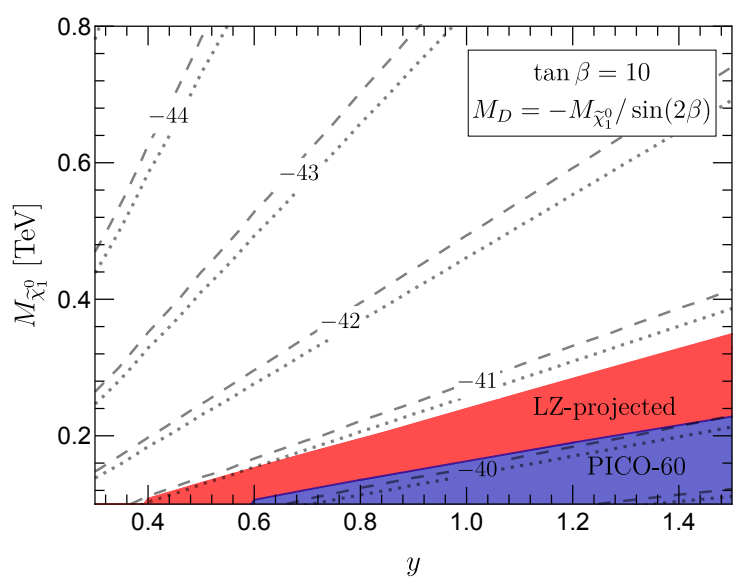

(b)

Figure 5. Spin-dependent DM-nucleon scattering cross-sections $\left(\sigma_{\mathrm{SD}}^{p, n}\right)$ in the DM mass $\left(M_{\widetilde{\chi}_{1}^{0}}\right)-$ Yukawa coupling $(y)$ plane, with all other parameters and conditions being the same as in figure 4 . The contours represent lines with fixed values of $\log _{10} \sigma_{\mathrm{SD}}^{p}$ (dashed) and $\log _{10} \sigma_{\mathrm{SD}}^{n}$ (dotted), with $\sigma_{\mathrm{SD}}^{p, n}$ expressed in $\mathrm{cm}^{2}$ units. The reach of the ongoing PICO-60 experiment (blue shaded) and the projected reach of the LZ experiment (red shaded) are also shown.

the future projection from LZ can probe DM masses upto $1560 \mathrm{GeV}$. For $\tan \beta=10$, the reach from PICO-60 is reduced to $230 \mathrm{GeV}$ and that of LZ to around $350 \mathrm{GeV}$.

Thus in the particular simple model adopted in this study the tree-level SD scattering has somewhat better prospects in probing the model parameter space, compared to the one-loop SI scattering rates. However, since the SI and SD rates probe the coupling of the DM particle to different sets of SM particles, both of them are necessary probes of the model, with combined experimental observations leading to a unified picture of the DM-nucleon effective couplings.

Before concluding, a special remark is in order. The search for missing particles, the potential DM candidates, at high-energy colliders is complementary to the DM direct detection. The charged and neutral dark sector states can be pair-produced in quarkantiquark annihilation via the $s$-channel $W^{ \pm}, Z$-boson exchange in the Drell-Yan process. These states, apart from the lightest neutral DM particle, would decay via electroweak interactions to final states containing $W$ and $Z$ bosons. Thus, multiple leptons and missing transverse momenta are the most promising channels to search for at hadron colliders, such as the LHC [44, 45], including its luminosity (HL-LHC) [46, 47], and possibly energy (HELHC) upgrades [48]. For small mass gaps between the charged and neutral dark sector particles, a likely scenario under our consideration, searches for disappearing tracks and displaced vertices are relevant [49]. For a detailed discussion of the LHC complementarity for DM search near the blind-spot region, we refer the reader to ref. [26]. On the other hand, the situation could be more optimistic if there are relatively light colored states (such as gluinos and squarks in SUSY), that could be copiously produced at hadron colliders and that could subsequently decay to the DM states resulting in large missing transverse momentum and multiple jets in the final state [50]. 


\section{Summary}

In this paper, we studied the NLO electroweak corrections to spin-independent dark matter nucleon scattering, in scenarios where the tree-level predictions for these rates are very small. Such small leading order rates are obtained generically in dark matter models where the DM state results from the mixing of electroweak singlet and doublet states, due to cancellations in DM coupling to the Higgs boson, which is the primary mediator of SI interactions for Majorana fermion WIMPs. A well-known example of these DM blind spots is the case of bino-Higgsino mixed DM in the MSSM. To understand the impact of radiative corrections to DM-nucleon scattering in such a setup, we adopted a simple model for DM with one Majorana fermion singlet, and two electroweak doublets with opposite hypercharge, the neutral components of which mix after electroweak symmetry breaking. This corresponds to the MSSM neutralino sector with all the sfermions, heavy scalars and wino decoupled.

We evaluated, adopting an on-shell renormalization scheme for the dark matter sector, the set of triangle and box diagrams for the radiative corrections to the DM-quark scalar effective operator, that could directly modify the predictions near the blind spots. We observed that the contribution to the DM-nucleon effective coupling $f_{N}$ from the triangle diagrams dominates near the tree-level blind spot, as the leading order contribution is vanishingly small in this region. As expected, the one-loop contributions "unblind" the tree-level blind spots, as seen in figure 2. Away from the blind-spot region, the one-loop electroweak effects are still found to be appreciable. For example, the triangle diagrams considered can shift the tree-level value of $f_{N}$ by upto $10 \%$. We also find that the box diagram contribution can become comparable to the triangles in some parameter regions. There are values of parameters around which both the triangle and the box contributions can also change sign, and therefore have their own blind spots.

Importantly, we always find a new blind spot at the NLO level where the sum of the tree-level and one-loop amplitudes go to zero. This leads to a shifted location for the blindspot point, the amount of the shift in the values of the doublet mass mixing parameter $M_{D}$ being almost linearly proportional to the value of the singlet mass $M_{S}$. This shift is found to be larger for large values of $\tan \beta$ (the ratio of the Yukawa couplings of the two doublets, $\left.y_{1} / y_{2}\right)$ and small values of $y\left(=\sqrt{y_{1}^{2}+y_{2}^{2}}\right)$, and can be around $\mathcal{O}(1 \%)$. These features are shown in figure 3.

On taking into account the impact of the radiative corrections to SI scattering, the prospects of testing such tree-level blind-spot scenarios in future multi-ton scale liquid Xenon experiments improve considerably. In particular, we find that for smaller values of $\tan \beta$, e.g., $\tan \beta=2, \sigma_{\mathrm{SI}}$ takes values in the range of about $10^{-47} \mathrm{~cm}^{2}$ to $10^{-50} \mathrm{~cm}^{2}$, for $M_{\widetilde{\chi}_{1}^{0}}$ values in the interval $100 \mathrm{GeV}-2 \mathrm{TeV}$, and coupling coefficient $y$ in the range $0.3-1.5$. For this range of couplings, the future projection of the LZ experiment is expected to probe a DM mass upto about $500 \mathrm{GeV}$, while the reach can be further extended by the DARWIN experiment upto a DM mass of $1250 \mathrm{GeV}$. On the other hand, for higher values of $\tan \beta$, as seen with $\tan \beta=10$, the expected cross-section is smaller, with a maximum of around $10^{-49} \mathrm{~cm}^{2}$, which may not be accessible to DARWIN. Thus, the small $\tan \beta$ scenario leads 
to similar $\sigma_{\mathrm{SI}}$ as in the case of wino-like real triplet $\mathrm{DM}$, while the intermediate $\tan \beta$ scenario predicts cross-sections similar to the case of Majorana Higgsino-like doublets. These results are presented in figure 4. On the other hand, as already examined in ref. [26], the SD scattering cross-sections may be observable in certain SI blind-spot regions. Thus, combined tests of both the SI one-loop predictions and the tree-level SD cross-sections are feasible, thereby probing all the relevant effective operators for DM-nucleon interaction.

With the increasing sensitivity of the dark matter direct detection experiments, resulting from the construction of bigger and ultra-low noise detectors, it is important to define benchmark targets for these near future multi-ton scale experiments. As we found in this study, higher order electroweak corrections to scenarios with mixed electroweak DM states present one such target, where the tree-level rates can be very small due to the vanishing of relevant DM effective couplings in certain parameter regions. In order to thoroughly probe interesting and well-motivated WIMP scenarios, it is therefore necessary to have theoretical predictions with increased accuracy that could match up to the future expected experimental precision.

\section{Acknowledgments}

We would like to thank Ayres Freitas for helpful discussions. This work is supported in part by the U.S. Department of Energy under grant No. DE-FG02-95ER40896 and by the PITT PACC. XW is also supported in part by an Andrew Mellon Predoctoral Fellowship from the School of Arts and Sciences at the University of Pittsburgh.

\section{A Details of on-shell renormalization scheme}

The Lagrangian of the DM sector in the mass basis can be written as

$$
\mathscr{L}=\overline{\widetilde{\chi}^{+}}\left(\not p P_{L}+\not p P_{R}-\eta M_{D}\right) \tilde{\chi}_{0}^{+}+\frac{1}{2} \bar{\chi}_{i}^{0}\left(\not p P_{L} \delta_{i j}+\not p P_{R} \delta_{i j}-\left[\mathbf{U}^{\top} \mathbf{M}_{\mathbf{N}} \mathbf{U}\right]_{i j}\right) \tilde{\chi}_{j}^{0},
$$

where $\eta$ is a phase factor and $i, j$ are summed over 1 to 3 . We specify the on-shell renormalization scheme adopted for the DM sector in the following.

According to the multiplicative renormalization procedure, we perform the following replacements of the parameters and the fields:

$$
\begin{array}{rlrl}
M_{S} & \rightarrow M_{S}+\delta M_{S}, & M_{D} & \rightarrow M_{D}+\delta M_{D}, \\
y_{1} & \rightarrow y_{1}+\delta y_{1}, & \rightarrow y_{2}+\delta y_{2}, \\
P_{L} \widetilde{\chi}^{+} & \rightarrow\left[1+\frac{1}{2} \delta Z_{\tilde{\chi}^{+}}^{L}\right]_{L} P_{L} \widetilde{\chi}^{+}, & P_{R} \widetilde{\chi}^{+} \rightarrow\left[1+\frac{1}{2} \delta Z_{\widetilde{\chi}^{+}}^{R}\right] P_{R} \widetilde{\chi}^{+}, \\
P_{L} \widetilde{\chi}_{i}^{0} & \rightarrow\left[\mathbb{1}+\frac{1}{2} \delta \mathbf{Z}_{\widetilde{\chi}^{0}}\right]_{i j} P_{L} \widetilde{\chi}_{j}^{0}, & P_{R} \widetilde{\chi}_{i}^{0} \rightarrow\left[\mathbb{1}+\frac{1}{2} \delta \mathbf{Z}_{\widetilde{\chi}^{0}}^{*}\right]_{i j} P_{R} \widetilde{\chi}_{j}^{0} .
\end{array}
$$


We note that the transformation matrix $\mathbf{U}$ is not renormalized in our scheme, so that, the mass matrix in the gauge basis $\mathbf{M}_{\mathbf{N}}$ is replaced by

$$
\mathbf{M}_{\mathbf{N}} \rightarrow \mathbf{M}_{\mathbf{N}}+\delta \mathbf{M}_{\mathbf{N}}=\mathbf{M}_{\mathbf{N}}+\left(\begin{array}{ccc}
\delta M_{S} & \delta \Delta_{2} & \delta \Delta_{1} \\
\delta \Delta_{2} & 0 & \delta M_{D} \\
\delta \Delta_{1} & \delta M_{D} & 0
\end{array}\right)
$$

where $\delta \Delta_{1,2}=\delta\left(y_{1,2} v / \sqrt{2}\right)$. Then the mass matrix in the mass basis can be expressed as

$$
\mathbf{M}_{\widetilde{\chi}^{0}} \rightarrow \mathbf{M}_{\widetilde{\chi}^{0}}+\delta \mathbf{M}_{\widetilde{\chi}^{0}}=\operatorname{diag}\left(M_{\widetilde{\chi}_{1}^{0}}, M_{\widetilde{\chi}_{2}^{0}}, M_{\widetilde{\chi}_{3}^{0}}\right)+\mathbf{U}^{\top} \delta \mathbf{M}_{\mathbf{N}} \mathbf{U}
$$

In the following, we use $\Sigma$ and $\hat{\Sigma}$ to denote un-renormalized and renormalized self-energies respectively. Decomposing into the following form

$$
\hat{\Sigma}(p)=\hat{\Sigma}^{L}\left(p^{2}\right) \not p P_{L}+\hat{\Sigma}^{R}\left(p^{2}\right) \not p P_{R}+\hat{\Sigma}^{S L}\left(p^{2}\right) P_{L}+\hat{\Sigma}^{S R}\left(p^{2}\right) P_{R},
$$

the renormalized self-energies of the charged and neutral states are given by

$$
\begin{aligned}
& \hat{\Sigma}_{\tilde{\chi}^{+}}^{L}\left(p^{2}\right)=\Sigma_{\tilde{\chi}^{+}}^{L}\left(p^{2}\right)+\frac{1}{2}\left(\delta Z_{\tilde{\chi}^{+}}^{L}+\delta Z_{\tilde{\chi}^{+}}^{L *}\right), \\
& \hat{\Sigma}_{\tilde{\chi}^{+}}^{R}\left(p^{2}\right)=\Sigma_{\tilde{\chi}^{+}}^{R}\left(p^{2}\right)+\frac{1}{2}\left(\delta Z_{\tilde{\chi}^{+}}^{R}+\delta Z_{\tilde{\chi}^{+}}^{R *}\right), \\
& \hat{\Sigma} \tilde{\chi}^{S L}\left(p^{2}\right)=\Sigma_{\tilde{\chi}^{+}}^{S L}\left(p^{2}\right)-\frac{1}{2}\left(M_{\tilde{\chi}^{+}} \delta Z_{\tilde{\chi}^{+}}^{L}+\delta Z_{\tilde{\chi}^{+}}^{R *} M_{\tilde{\chi}^{+}}+2 \delta M_{\tilde{\chi}^{+}}\right), \\
& \hat{\Sigma}_{\tilde{\chi}^{+}}^{S R}\left(p^{2}\right)=\Sigma_{\tilde{\chi}^{+}}^{S R}\left(p^{2}\right)-\frac{1}{2}\left(M_{\tilde{\chi}^{+}} \delta Z_{\tilde{\chi}^{+}}^{R}+\delta Z_{\tilde{\chi}^{+}}^{L *} M_{\tilde{\chi}^{+}}+2 \delta M_{\tilde{\chi}^{+}}^{*}\right), \\
& {\left[\hat{\Sigma}_{\tilde{\chi}^{0}}^{L}\left(p^{2}\right)\right]_{i j}=\left[\Sigma_{\widetilde{\chi}^{0}}^{L}\left(p^{2}\right)\right]_{i j}+\frac{1}{2}\left[\delta \mathbf{Z}_{\tilde{\chi}^{0}}+\delta \mathbf{Z}_{\widetilde{\chi}^{0}}^{\dagger}\right]_{i j},} \\
& {\left[\hat{\Sigma}_{\widetilde{\chi}^{0}}^{R}\left(p^{2}\right)\right]_{i j}=\left[\Sigma_{\widetilde{\chi}^{0}}^{R}\left(p^{2}\right)\right]_{i j}+\frac{1}{2}\left[\delta \mathbf{Z}_{\widetilde{\chi}^{0}}^{*}+\delta \mathbf{Z}_{\widetilde{\chi}^{0}}^{\top}\right]_{i j},} \\
& {\left[\hat{\Sigma}_{\widetilde{\chi}^{0}}^{S L}\left(p^{2}\right)\right]_{i j}=\left[\sum_{\widetilde{\chi}^{0}}^{S L}\left(p^{2}\right)\right]_{i j}-\frac{1}{2}\left[\mathbf{M}_{\widetilde{\chi}^{0}} \delta \mathbf{Z}_{\widetilde{\chi}^{0}}+\delta \mathbf{Z}_{\widetilde{\chi}^{0}}^{\top} \mathbf{M}_{\widetilde{\chi}^{0}}+2 \delta \mathbf{M}_{\widetilde{\chi}^{0}}\right]_{i j},}
\end{aligned}
$$

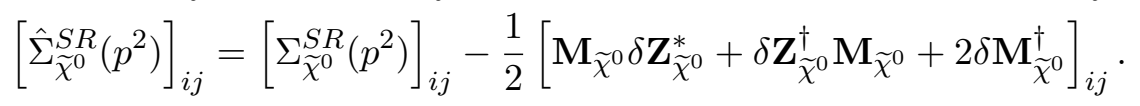

We choose the on-shell renormalization scheme by imposing (for $i, j=1,2,3$ )

$$
\begin{aligned}
& {\left.\left[\widetilde{\operatorname{Re}} \hat{\Sigma}_{\widetilde{\chi}^{+}}(p)\right] \widetilde{\chi}^{+}(p)\right|_{p^{2}=M_{\tilde{\chi}^{+}}^{2}}=0, \quad \lim _{p^{2} \rightarrow M_{\tilde{\chi}^{+}}^{2}} \frac{1}{\not p-M_{\widetilde{\chi}^{+}}}\left[\widetilde{\operatorname{Re}} \hat{\Sigma}_{\widetilde{\chi}^{+}}(p)\right] \widetilde{\chi}^{+}(p)=0,} \\
& {\left.\left[\widetilde{\operatorname{Re}} \hat{\Sigma}_{\widetilde{\chi}^{0}}(p)\right]_{i j} \widetilde{\chi}_{j}^{0}(p)\right|_{p^{2}=M_{\widetilde{\chi}_{j}^{0}}^{2}}=0, \quad \lim _{p^{2} \rightarrow M_{\widetilde{\chi}_{i}^{0}}^{2}} \frac{1}{\not p-M_{\widetilde{\chi}_{i}^{0}}}\left[\widetilde{\operatorname{Re}} \hat{\Sigma}_{\widetilde{\chi}^{0}}(p)\right]_{i i} \widetilde{\chi}_{i}^{0}(p)=0,}
\end{aligned}
$$

where $\widetilde{R e}$ takes only the real part of the loop integrals appearing in the self energies but not of the mixing matrix elements or couplings appearing therein. We further fix the imaginary parts of the wave-function renormalization constants by choosing

$$
\operatorname{Im}\left[\delta Z_{\widetilde{\chi}^{+}}^{L}\right]=\operatorname{Im}\left[\delta Z_{\widetilde{\chi}^{+}}^{R}\right]=\operatorname{Im}\left[\delta \mathbf{Z}_{\widetilde{\chi}^{0}}\right]_{i i}=0 .
$$


Thus, eqs. (A.17)-(A.19) yield the counterterms

$$
\begin{aligned}
& \delta Z_{\tilde{\chi}^{+}}^{L}=-\Sigma_{\tilde{\chi}^{+}}^{L}\left(M_{\tilde{\chi}^{+}}^{2}\right)-M_{\tilde{\chi}^{+}}^{2}\left[\Sigma_{\tilde{\chi}^{+}}^{L^{\prime}}\left(M_{\tilde{\chi}^{+}}^{2}\right)+\Sigma_{\tilde{\chi}^{+}}^{R^{\prime}}\left(M_{\tilde{\chi}^{+}}^{2}\right)\right] \\
& -M_{\tilde{\chi}^{+}}\left[\Sigma_{\tilde{\chi}^{+}}^{S L^{\prime}}\left(M_{\tilde{\chi}^{+}}^{2}\right)+\Sigma_{\tilde{\chi}^{+}}^{S R^{\prime}}\left(M_{\tilde{\chi}^{+}}^{2}\right)\right], \\
& \delta Z_{\tilde{\chi}^{+}}^{R}=-\Sigma_{\tilde{\chi}^{+}}^{R}\left(M_{\tilde{\chi}^{+}}^{2}\right)-M_{\tilde{\chi}^{+}}^{2}\left[\Sigma_{\tilde{\chi}^{+}}^{L^{\prime}}\left(M_{\tilde{\chi}^{+}}^{2}\right)+\Sigma_{\tilde{\chi}^{+}}^{R^{\prime}}\left(M_{\tilde{\chi}^{+}}^{2}\right)\right] \\
& -M_{\tilde{\chi}^{+}}\left[\Sigma_{\tilde{\chi}^{+}}^{S L^{\prime}}\left(M_{\tilde{\chi}^{+}}^{2}\right)+\Sigma_{\tilde{\chi}^{+}}^{S R^{\prime}}\left(M_{\tilde{\chi}^{+}}^{2}\right)\right] \text {, } \\
& \delta M_{D}=\eta^{*} \delta M_{\tilde{\chi}^{+}}=\frac{\eta^{*}}{2} M_{\tilde{\chi}^{+}}\left[\Sigma_{\tilde{\chi}^{+}}^{L}\left(M_{\tilde{\chi}^{+}}^{2}\right)+\Sigma_{\widetilde{\chi}^{+}}^{R}\left(M_{\tilde{\chi}^{+}}^{2}\right)\right]+\eta^{*} \Sigma_{\tilde{\chi}^{+}}^{S L}\left(M_{\tilde{\chi}^{+}}^{2}\right), \\
& {\left[\delta \mathbf{Z}_{\widetilde{\chi}^{0}}\right]_{i i}=-\frac{1}{2}\left[\Sigma_{\widetilde{\chi}^{0}}^{L}\left(M_{\widetilde{\chi}_{i}^{0}}^{2}\right)+\Sigma_{\widetilde{\chi}_{i}^{0}}^{R}\left(M_{\widetilde{\chi}_{i}^{0}}^{2}\right)\right]_{i i}} \\
& -M_{\widetilde{\chi}_{i}^{0}}^{2}\left[\Sigma_{\widetilde{\chi}^{0}}^{L^{\prime}}\left(M_{\widetilde{\chi}_{i}^{0}}^{2}\right)+\Sigma_{\widetilde{\chi}^{+}}^{R^{\prime}}\left(M_{\widetilde{\chi}_{i}^{0}}^{2}\right)\right]_{i i}-M_{\widetilde{\chi}_{i}^{0}}\left[\sum_{\widetilde{\chi}^{0}}^{S L^{\prime}}\left(M_{\widetilde{\chi}_{i}^{0}}^{2}\right)+\sum_{\widetilde{\chi}^{0}}^{S R^{\prime}}\left(M_{\widetilde{\chi}_{i}^{0}}^{2}\right)\right]_{i i}, \\
& {\left[\delta \mathbf{Z}_{\widetilde{\chi}^{0}}\right]_{i j}=\frac{2}{M_{\widetilde{\chi}_{i}^{0}}^{2}-M_{\widetilde{\chi}_{j}^{0}}^{2}}\left[M_{\widetilde{\chi}_{j}^{0}}^{2} \Sigma_{\widetilde{\chi}^{0}}^{L}\left(M_{\widetilde{\chi}_{j}^{0}}^{2}\right)+M_{\widetilde{\chi}_{i}^{0}} M_{\widetilde{\chi}_{j}^{0}} \Sigma_{\widetilde{\chi}^{0}}^{R}\left(M_{\widetilde{\chi}_{j}^{0}}^{2}\right)+M_{\widetilde{\chi}_{i}^{0}} \Sigma_{\widetilde{\chi}^{0}}^{S L}\left(M_{\widetilde{\chi}_{j}^{0}}^{2}\right)\right.} \\
& \left.+M_{\widetilde{\chi}_{j}^{0}} \sum_{\widetilde{\chi}^{0}}^{S R}\left(M_{\widetilde{\chi}_{j}^{0}}^{2}\right)-M_{\widetilde{\chi}_{i}^{0}} \delta \mathbf{M}_{\widetilde{\chi}^{0}}-M_{\widetilde{\chi}_{j}^{0}} \delta \mathbf{M}_{\widetilde{\chi}^{0}}^{\dagger}\right]_{i j}, \quad \text { for } i \neq j, \\
& {\left[\delta \mathbf{M}_{\widetilde{\chi}^{0}}\right]_{i i}=\frac{1}{2} M_{\widetilde{\chi}_{i}^{0}}\left[\Sigma_{\widetilde{\chi}^{0}}^{L}\left(M_{\widetilde{\chi}_{i}^{0}}^{2}\right)+\Sigma_{\widetilde{\chi}^{0}}^{R}\left(M_{\widetilde{\chi}_{i}^{0}}^{2}\right)\right]_{i i}+\left[\sum_{\widetilde{\chi}^{0}}^{S L}\left(M_{\widetilde{\chi}_{i}^{0}}^{2}\right)\right]_{i i},}
\end{aligned}
$$

where $\Sigma^{\prime}\left(p^{2}\right)$ is the derivative of the self-energy $\Sigma^{\prime}\left(p^{2}\right)=\partial \Sigma\left(p^{2}\right) / \partial p^{2}$. All the unrenormalized self-energies $\Sigma$ in eqs. (A.20)-(A.25) should be understood as $\widetilde{\operatorname{Re}} \Sigma$. The counterterms $\delta \Delta_{1}, \delta \Delta_{2}$ and $\delta M_{S}$ in eq. (A.6) are then fixed by solving the equations

$$
\left[\delta \mathbf{M}_{\widetilde{\chi}^{0}}\right]_{i i}=\left[\mathbf{U}^{\top} \delta \mathbf{M}_{\mathbf{N}} \mathbf{U}\right]_{i i} .
$$

The relevant counterterms to the DM-Higgs coupling can be expressed as

$$
\begin{aligned}
\delta \Gamma^{\mathrm{ct}}\left(\overline{\widetilde{\chi}}_{1}^{0}, \widetilde{\chi}_{1}^{0}, h\right)= & {\left[\delta \mathbf{S}^{L}+\frac{1}{2} \delta \mathbf{Z}_{\widetilde{\chi}^{0}}^{\top} \mathbf{S}^{L}+\frac{1}{2} \mathbf{S}^{L} \delta \mathbf{Z}_{\widetilde{\chi}^{0}}+\frac{1}{2} \mathbf{S}^{L} \delta Z_{h}\right]_{11} P_{L} } \\
& +\left[\delta \mathbf{S}^{R}+\frac{1}{2} \delta \mathbf{Z}_{\widetilde{\chi}^{0}}^{\dagger} \mathbf{S}^{R}+\frac{1}{2} \mathbf{S}^{R} \delta \mathbf{Z}_{\widetilde{\chi}^{0}}^{*}+\frac{1}{2} \mathbf{S}^{R} \delta Z_{h}\right]_{11} P_{R} .
\end{aligned}
$$

where

$$
\begin{aligned}
{\left[\mathbf{S}^{L}\right]_{i i} } & =-\frac{y_{1}}{\sqrt{2}} U_{3 i} U_{1 i}-\frac{y_{2}}{\sqrt{2}} U_{2 i} U_{1 i}, \\
{\left[\mathbf{S}^{L}\right]_{i j} } & =-\frac{y_{1}}{\sqrt{2}} U_{3 i} U_{1 j}-\frac{y_{2}}{\sqrt{2}} U_{2 i} U_{1 j}+(i \leftrightarrow j), \quad \text { for } i \neq j, \\
\mathbf{S}_{R} & =\mathbf{S}_{L}^{\dagger} .
\end{aligned}
$$

The counterterms $\delta y_{1}$ and $\delta y_{2}$ are related to $\delta \Delta_{1}$ and $\delta \Delta_{2}$ through the relations

$$
\delta y_{1}=\sqrt{2} \frac{\delta \Delta_{1}}{v}-y_{1} \frac{\delta v}{v}, \quad \delta y_{2}=\sqrt{2} \frac{\delta \Delta_{2}}{v}-y_{2} \frac{\delta v}{v},
$$

with $\delta v$ and $\delta Z_{h}$ calculated in the on-shell scheme following the conventions in ref. [51]. 


\section{B DM-nucleon scattering: computational framework}

In this appendix, we briefly review the formalism adopted for computing the DM-nucleon scattering cross-sections [1], and the values of the relevant nuclear matrix elements used. The effective interactions of a non-relativistic Majorana WIMP $X$ with light quarks and gluons are given as

$$
\mathscr{L}_{\mathrm{eff}}=\sum_{q=u, d, s}\left(d_{q} \bar{X} \gamma^{\mu} \gamma^{5} X \bar{q} \gamma_{\mu} \gamma^{5} q+f_{q} m_{q} \bar{X} X \bar{q} q\right)+f_{G} \bar{X} X \frac{\alpha_{s}}{\pi} G_{\mu \nu}^{a} G^{a \mu \nu},
$$

where, $G_{\mu \nu}^{a}$ is the gluon field strength tensor and $\alpha_{S}$ is the strong coupling constant. Here, the operator involving axial-vector currents of the DM and the quark fields leads to spindependent interactions, while the other two operator structures lead to spin-independent scattering with nuclei.

To begin with, we define the matrix element (ME) of the scalar operator $\bar{q} q$ between nucleon states $N$ (where $N$ is either a proton or a neutron) as follows:

$$
\left\langle N\left|m_{q} \bar{q} q\right| N\right\rangle \equiv f_{T q}^{N} m_{N} .
$$

The corresponding ME of the gluon operator can be obtained by using the trace of the energy momentum tensor $T_{\mu}^{\mu}$, which is given by

$$
T_{\mu}^{\mu}=\sum_{q=u, d, s} m_{q} \bar{q} q+\sum_{Q=b, c, t} m_{Q} \bar{Q} Q-\frac{7 \alpha_{s}}{8 \pi} G G .
$$

Here, we have used the shorthand $G G$ to stand for $G_{\mu \nu}^{a} G^{a \mu \nu}$. Utilizing the fact that

$$
\left\langle N\left|T_{\mu}^{\mu}\right| N\right\rangle \equiv m_{N}
$$

where, $m_{N}$ is the nucleon mass, and by integrating out the heavy quarks using

$$
\left\langle N\left|m_{Q} \bar{Q} Q\right| N\right\rangle=\left\langle N\left|-\frac{\alpha_{s}}{12 \pi} G G\right| N\right\rangle,
$$

we obtain the ME of the gluon operator

$$
\left\langle N\left|\frac{\alpha_{s}}{\pi} G G\right| N\right\rangle=-\frac{8}{9} m_{N} f_{T G}^{N}
$$

Here, $f_{T G}^{N}$ is related to $f_{T q}^{N}$ as

$$
f_{T G}^{N} \equiv 1-\sum_{q=u, d, s} f_{T q}^{N} .
$$

Similarly, the nucleon ME of the axial-vector quark current is defined as

$$
\left\langle N\left|\bar{q} \gamma_{\mu} \gamma^{5} q\right| N\right\rangle \equiv 2 s_{\mu} \Delta q_{N},
$$

where $s_{\mu}$ is the nucleon spin. Combining these results, the effective interaction of Majorana WIMPs with nucleons is given by

$$
\mathscr{L}_{\text {eff }}=\sum_{N=n, p}\left(f_{N} \bar{X} X \bar{N} N+a_{N} \bar{X} \gamma^{\mu} \gamma^{5} X \bar{N} \gamma_{\mu} \gamma^{5} N\right),
$$


with the Wilson co-efficients,

$$
f_{N} / m_{N}=\sum_{q=u, d, s} f_{q} f_{T q}^{N}-\frac{8}{9} f_{G} f_{T G}^{N} \quad \text { and } \quad a_{N}=\sum_{q=u, d, s} d_{q} \Delta q_{N} .
$$

For our computations, we adopt the following values of the nuclear matrix elements for proton: $f_{T u}^{p}=0.0153, f_{T d}^{p}=0.0191$, and $f_{T s}^{p}=0.0447$, where we have used the lattice results for the strange quark content of the nucleon [52-54]. For spin-dependent scattering, we use the following inputs: $\Delta u_{p}=0.842, \Delta d_{p}=-0.427$, and $\Delta s_{p}=-0.085$ [54].

\section{Mapping the singlet-doublet model to MSSM}

The analysis presented in section 2 can be translated to the neutralino sector in the minimal supersymmetric standard model (MSSM), with the wino state decoupled. In such a scenario, the neutralino mass matrix in the basis $\left(\widetilde{B}, \widetilde{H}_{d}^{0}, \widetilde{H}_{u}^{0}\right)$ is given by

$$
M_{N}=\left(\begin{array}{ccc}
M_{1} & -M_{Z} s_{W} \cos \beta & M_{Z} s_{W} \sin \beta \\
-M_{Z} s_{W} \cos \beta & 0 & -\mu \\
M_{Z} s_{W} \sin \beta & -\mu & 0
\end{array}\right)
$$

The phenomenology of tree-level spin-independent DM-quark interactions is then similar to what we obtained for the singlet-doublet model, with the following mapping between the couplings,

$$
y \rightarrow-\sqrt{2} \frac{M_{Z} s_{W}}{v} .
$$

The singlet and doublet fermion mass parameters $M_{S}$ and $M_{D}$ are replaced by the bino and Higgsino mass parameters, $M_{1}$ and $\mu$, respectively. The coupling of the lighter Higgs boson state to the lightest neutralino is then given by

$$
g_{h \chi_{1} \chi_{1}}^{0} \simeq \frac{e M_{Z} \tan \theta_{W}}{\mu^{2}-M_{1}^{2}}\left(M_{1}+\mu \sin (2 \beta)\right)
$$

In the MSSM, we also have the following DM coupling to the heavier CP-even Higgs boson

$$
g_{H \chi_{1} \chi_{1}}^{0} \simeq-\frac{e M_{Z} \tan \theta_{W}}{\mu^{2}-M_{1}^{2}} \mu \cos (2 \beta) .
$$

Combining with the Higgs-quark Yukawa couplings and taking the alignment limit, at the leading order the DM-quark scalar effective couplings are then obtained to be

$$
f_{u}=-\frac{g_{h \chi_{1} \chi_{1}}^{0}}{v m_{h}^{2}}+\frac{g_{H \chi_{1} \chi_{1}}^{0}}{v m_{H}^{2}} \cot \beta, \quad f_{d}=-\frac{g_{h \chi_{1} \chi_{1}}^{0}}{v m_{h}^{2}}-\frac{g_{H \chi_{1} \chi_{1}}^{0}}{v m_{H}^{2}} \tan \beta .
$$

In the scenario with the heavy Higgs decoupled, we can now obtain the SI blind-spot condition for MSSM:

$$
M_{1}+\mu \sin (2 \beta)=0
$$

with $\operatorname{sgn}\left(M_{1} / \mu\right)=-1$. 
Open Access. This article is distributed under the terms of the Creative Commons Attribution License (CC-BY 4.0), which permits any use, distribution and reproduction in any medium, provided the original author(s) and source are credited.

\section{References}

[1] G. Jungman, M. Kamionkowski and K. Griest, Supersymmetric dark matter, Phys. Rept. 267 (1996) 195 [hep-ph/9506380] [INSPIRE].

[2] G. Bertone, D. Hooper and J. Silk, Particle dark matter: Evidence, candidates and constraints, Phys. Rept. 405 (2005) 279 [hep-ph/0404175] [INSPIRE].

[3] XENON collaboration, Dark Matter Search Results from a One Ton-Year Exposure of XENON1T, Phys. Rev. Lett. 121 (2018) 111302 [arXiv:1805.12562] [INSPIRE].

[4] LUX collaboration, Results from a search for dark matter in the complete LUX exposure, Phys. Rev. Lett. 118 (2017) 021303 [arXiv:1608.07648] [INSPIRE].

[5] PandaX-II collaboration, Dark Matter Results From 54-Ton-Day Exposure of PandaX-II Experiment, Phys. Rev. Lett. 119 (2017) 181302 [arXiv:1708.06917] [INSPIRE].

[6] M. Cirelli, N. Fornengo and A. Strumia, Minimal dark matter, Nucl. Phys. B 753 (2006) 178 [hep-ph/0512090] [INSPIRE].

[7] M. Cirelli, A. Strumia and M. Tamburini, Cosmology and Astrophysics of Minimal Dark Matter, Nucl. Phys. B 787 (2007) 152 [arXiv:0706.4071] [INSPIRE].

[8] J. Hisano, S. Matsumoto, M.M. Nojiri and O. Saito, Direct detection of the Wino and Higgsino-like neutralino dark matters at one-loop level, Phys. Rev. D 71 (2005) 015007 [hep-ph/0407168] [INSPIRE].

[9] J. Hisano, K. Ishiwata and N. Nagata, A complete calculation for direct detection of Wino dark matter, Phys. Lett. B 690 (2010) 311 [arXiv: 1004.4090] [INSPIRE].

[10] J. Hisano, K. Ishiwata, N. Nagata and T. Takesako, Direct Detection of Electroweak-Interacting Dark Matter, JHEP 07 (2011) 005 [arXiv:1104.0228] [INSPIRE].

[11] R.J. Hill and M.P. Solon, WIMP-nucleon scattering with heavy WIMP effective theory, Phys. Rev. Lett. 112 (2014) 211602 [arXiv:1309.4092] [INSPIRE].

[12] J. Hisano, K. Ishiwata and N. Nagata, QCD Effects on Direct Detection of Wino Dark Matter, JHEP 06 (2015) 097 [arXiv: 1504.00915] [INSPIRE].

[13] L.E. Strigari, Neutrino Coherent Scattering Rates at Direct Dark Matter Detectors, New J. Phys. 11 (2009) 105011 [arXiv: 0903.3630] [INSPIRE].

[14] J. Billard, L. Strigari and E. Figueroa-Feliciano, Implication of neutrino backgrounds on the reach of next generation dark matter direct detection experiments, Phys. Rev. D 89 (2014) 023524 [arXiv: 1307.5458] [inSPIRE].

[15] P. Grothaus, M. Fairbairn and J. Monroe, Directional Dark Matter Detection Beyond the Neutrino Bound, Phys. Rev. D 90 (2014) 055018 [arXiv: 1406.5047] [INSPIRE].

[16] C.A.J. O'Hare, A.M. Green, J. Billard, E. Figueroa-Feliciano and L.E. Strigari, Readout strategies for directional dark matter detection beyond the neutrino background, Phys. Rev. D 92 (2015) 063518 [arXiv: 1505.08061] [INSPIRE].

[17] C. Cheung, L.J. Hall, D. Pinner and J.T. Ruderman, Prospects and Blind Spots for Neutralino Dark Matter, JHEP 05 (2013) 100 [arXiv:1211.4873] [INSPIRE]. 
[18] C. Cheung and D. Sanford, Simplified Models of Mixed Dark Matter, JCAP 02 (2014) 011 [arXiv: 1311.5896] [INSPIRE].

[19] J.L. Feng, J. Kumar and D. Sanford, Xenophobic Dark Matter, Phys. Rev. D 88 (2013) 015021 [arXiv: 1306.2315] [inSPIRE].

[20] A. Dedes and D. Karamitros, Doublet-Triplet Fermionic Dark Matter, Phys. Rev. D 89 (2014) 115002 [arXiv: 1403.7744] [INSPIRE].

[21] P. Huang and C.E.M. Wagner, Blind Spots for neutralino Dark Matter in the MSSM with an intermediate $m_{A}$, Phys. Rev. D 90 (2014) 015018 [arXiv:1404.0392] [INSPIRE].

[22] A. Crivellin, M. Hoferichter, M. Procura and L.C. Tunstall, Light stops, blind spots and isospin violation in the MSSM, JHEP 07 (2015) 129 [arXiv: 1503.03478] [INSPIRE].

[23] A. Freitas, S. Westhoff and J. Zupan, Integrating in the Higgs Portal to Fermion Dark Matter, JHEP 09 (2015) 015 [arXiv:1506.04149] [INSPIRE].

[24] M. Badziak, M. Olechowski and P. Szczerbiak, Blind spots for neutralino dark matter in the NMSSM, JHEP 03 (2016) 179 [arXiv:1512.02472] [INSPIRE].

[25] S. Banerjee, S. Matsumoto, K. Mukaida and Y.-L.S. Tsai, WIMP Dark Matter in a Well-Tempered Regime: A case study on Singlet-Doublets Fermionic WIMP, JHEP 11 (2016) 070 [arXiv : 1603.07387] [INSPIRE].

[26] T. Han, F. Kling, S. Su and Y. Wu, Unblinding the dark matter blind spots, JHEP 02 (2017) 057 [arXiv: 1612. 02387] [INSPIRE].

[27] S. Baum, M. Carena, N.R. Shah and C.E.M. Wagner, Higgs portals for thermal Dark Matter. EFT perspectives and the NMSSM, JHEP 04 (2018) 069 [arXiv:1712.09873] [INSPIRE].

[28] R. Mahbubani and L. Senatore, The Minimal model for dark matter and unification, Phys. Rev. D 73 (2006) 043510 [hep-ph/0510064] [INSPIRE].

[29] F. D'Eramo, Dark matter and Higgs boson physics, Phys. Rev. D 76 (2007) 083522 [arXiv:0705.4493] [INSPIRE].

[30] T. Hahn, Generating Feynman diagrams and amplitudes with FeynArts 3, Comput. Phys. Commun. 140 (2001) 418 [hep-ph/0012260] [INSPIRE].

[31] V. Shtabovenko, R. Mertig and F. Orellana, New Developments in FeynCalc 9.0, Comput. Phys. Commun. 207 (2016) 432 [arXiv: 1601.01167] [INSPIRE].

[32] R. Mertig, M. Böhm and A. Denner, FEYN CALC: Computer algebraic calculation of Feynman amplitudes, Comput. Phys. Commun. 64 (1991) 345 [InSPIRE].

[33] A. Denner, S. Dittmaier and L. Hofer, Collier: a fortran-based Complex One-Loop LIbrary in Extended Regularizations, Comput. Phys. Commun. 212 (2017) 220 [arXiv:1604.06792] [INSPIRE].

[34] A. Denner and S. Dittmaier, Reduction of one loop tensor five point integrals, Nucl. Phys. B 658 (2003) 175 [hep-ph/0212259] [INSPIRE].

[35] A. Denner and S. Dittmaier, Reduction schemes for one-loop tensor integrals, Nucl. Phys. B 734 (2006) 62 [hep-ph/0509141] [INSPIRE].

[36] A. Denner and S. Dittmaier, Scalar one-loop 4-point integrals, Nucl. Phys. B 844 (2011) 199 [arXiv: 1005.2076] [INSPIRE].

[37] N. Anand, A.L. Fitzpatrick and W.C. Haxton, Weakly interacting massive particle-nucleus elastic scattering response, Phys. Rev. C 89 (2014) 065501 [arXiv:1308.6288] [INSPIRE]. 
[38] LUX-ZEPLIN collaboration, Projected WIMP Sensitivity of the LUX-ZEPLIN (LZ) Dark Matter Experiment, arXiv:1802.06039 [INSPIRE].

[39] DARWIN collaboration, DARWIN: towards the ultimate dark matter detector, JCAP 11 (2016) 017 [arXiv : 1606.07001] [INSPIRE].

[40] L. Calibbi, A. Mariotti and P. Tziveloglou, Singlet-Doublet Model: Dark matter searches and LHC constraints, JHEP 10 (2015) 116 [arXiv:1505.03867] [INSPIRE].

[41] PICO collaboration, Dark Matter Search Results from the PICO-2L $C_{3} F_{8}$ Bubble Chamber, Phys. Rev. Lett. 114 (2015) 231302 [arXiv: 1503.00008] [INSPIRE].

[42] PICO collaboration, Dark matter search results from the PICO-60 $\mathrm{CF}_{3} I$ bubble chamber, Phys. Rev. D 93 (2016) 052014 [arXiv: 1510.07754] [INSPIRE].

[43] PICO collaboration, Dark Matter Search Results from the PICO-60 $C_{3} F_{8}$ Bubble Chamber, Phys. Rev. Lett. 118 (2017) 251301 [arXiv:1702.07666] [INSPIRE].

[44] ATLAS collaboration, Search for electroweak production of supersymmetric particles in final states with two or three leptons at $\sqrt{s}=13 \mathrm{TeV}$ with the ATLAS detector, Eur. Phys. J. C 78 (2018) 995 [arXiv: 1803.02762] [INSPIRE].

[45] CMS collaboration, Search for electroweak production of charginos and neutralinos in multilepton final states in proton-proton collisions at $\sqrt{s}=13 \mathrm{TeV}$, JHEP 03 (2018) 166 [arXiv: 1709.05406] [INSPIRE].

[46] ATLAS collaboration, Search for Supersymmetry at the high luminosity LHC with the ATLAS experiment, ATL-PHYS-PUB-2014-010.

[47] CMS collaboration, Supersymmetry discovery potential in future LHC and HL-LHC running with the CMS detector, CMS-PAS-SUS-14-012.

[48] A. Aboubrahim and P. Nath, Supersymmetry at a 28 TeV hadron collider: HE-LHC, Phys. Rev. D 98 (2018) 015009 [arXiv: 1804.08642] [INSPIRE].

[49] T. Han, S. Mukhopadhyay and X. Wang, Electroweak Dark Matter at Future Hadron Colliders, Phys. Rev. D 98 (2018) 035026 [arXiv: 1805.00015] [InSPIRE].

[50] ATLAS collaboration, Search for squarks and gluinos in final states with jets and missing transverse momentum at $\sqrt{s}=13 \mathrm{TeV}$ with the ATLAS detector, Eur. Phys. J. C 76 (2016) 392 [arXiv: 1605.03814] [INSPIRE].

[51] A. Denner, Techniques for calculation of electroweak radiative corrections at the one loop level and results for W physics at LEP-200, Fortsch. Phys. 41 (1993) 307 [arXiv:0709.1075] [INSPIRE].

[52] L. Álvarez-Ruso, T. Ledwig, J. Martin Camalich and M.J. Vicente-Vacas, Nucleon mass and pion-nucleon sigma term from a chiral analysis of lattice QCD data, Phys. Rev. D 88 (2013) 054507 [arXiv: 1304.0483 ] [INSPIRE].

[53] P. Junnarkar and A. Walker-Loud, Scalar strange content of the nucleon from lattice QCD, Phys. Rev. D 87 (2013) 114510 [arXiv:1301.1114] [INSPIRE].

[54] G. Bélanger, F. Boudjema, A. Pukhov and A. Semenov, MicrOMEGAs_3: A program for calculating dark matter observables, Comput. Phys. Commun. 185 (2014) 960 [arXiv: 1305.0237] [INSPIRE]. 